\title{
Light scattering by ultrasonically-controlled small particles: system design, calibration, and measurement results
}

\author{
Ivan Kassamakov a,b*, Göran Maconi ${ }^{\mathrm{a}}$, Antti Penttiläa ${ }^{\mathrm{a}}$, Petteri Helander ${ }^{\mathrm{a}}$, Maria Gritsevich ${ }^{\mathrm{a}, \mathrm{c}}$, Tuomas \\ Puranen ${ }^{\mathrm{a}}$, Ari Salmi ${ }^{\mathrm{a}}$, Edward Hæggström ${ }^{\mathrm{a}}$ and Karri Muinonen ${ }^{\mathrm{a}, \mathrm{d}}$
}

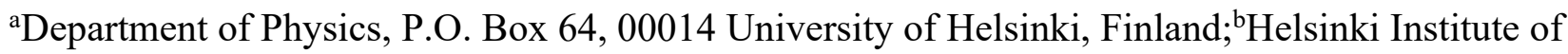
Physics, P.O. Box 64, 00014 University of Helsinki, Finland;'Institute of Physics and Technology, Ural Federal University, 620002 Ekaterinburg, Russia; ${ }^{d}$ Finnish Geospatial Research Institute FGI, Geodeetinrinne 2, 02430 Masala, Finland

\begin{abstract}
We present the design of a novel scatterometer for precise measurement of the angular Mueller matrix profile of a mm- to $\mu \mathrm{m}$-sized sample held in place by sound. The scatterometer comprises a tunable multimode Argon-krypton laser (with possibility to set 1 of the 12 wavelengths in visible range), linear polarizers, a reference photomultiplier tube (PMT) for monitoring the beam intensity, and a micro-PMT module mounted radially towards the sample at an adjustable radius. The measurement angle is controlled by a motor-driven rotation stage with an accuracy of $15^{\prime}$. The system is fully automated using LabVIEW, including the FPGA-based data acquisition and the instrument's user interface. The calibration protocol ensures accurate measurements by using a control sphere sample (diameter $3 \mathrm{~mm}$, refractive index of 1.5) fixed first on a static holder followed by accurate multi-wavelength measurements of the same sample levitated ultrasonically.

To demonstrate performance of the scatterometer, we conducted detailed measurements of light scattered by a particle derived from the Chelyabinsk meteorite, as well as planetary analogue materials. The measurements are the first of this kind, since they are obtained using controlled spectral angular scattering including linear polarization effects, for arbitrary shaped objects. Thus, our novel approach permits a non-destructive, disturbance-free measurement with control of the orientation and location of the scattering object.
\end{abstract}

Keywords: light scattering, polarized reflectance, calibration, optical properties

\section{INTRODUCTION}

Nondestructive measurements preserve the sample, which permits characterizing unique samples like samples returned from Solar System objects in different laboratories. Although characterization usually takes place in high quality cleanroom facilities, presence of the sample-holder in the experiment may drastically affect the results, especially in the case of $\mu \mathrm{m}$-sized samples. Another important aspect is preservation of the sample in its original condition, which in many cases is challenging. In this paper we present the design of a novel scatterometer for precise measurement of the angular Mueller matrix profile of $\mathrm{mm}$ - to $\mu \mathrm{m}$-sized samples held in place in controllable manner by sound.

Electromagnetic scattering is a fundamental physical process that allows to determine optical characteristics and to estimate the shape of an object studied remotely. This possibility is enhanced by obtaining the light-scattering response at multiple wavelengths and viewing geometries, by considering a wider range of the phase angle (the angle between the incident light and the light reflected from the object) during the measurements. Experimentally derived data for Mueller matrices of a sample can be used as input for larger scale scattering simulations, without using idealized model for single-particle properties. Scattering theory already finds use in the creation of atmospheric models, catalytic agents, next-generation capacitors, medical diagnostic toolkits, and drug carriers. Additionally, scattering-based imaging could create a pathway towards super-resolution imaging.

*email: ivan.kassamakov@helsinki.fi 
One of the first scatterometry setups for small particle characterization was built in Arizona (Department of Physics, University of Arizona, Tucson) ${ }^{1}$. The system was used to characterize 110-nm diameter latex spheres. The light source was a high-pressure Hg lamp (Osram HBO 100). To acquire all the elements of the Mueller matrix, polarized light was used. Later on, another system was built at the Department of Physics and Astronomy, Free University, Amsterdam, the Netherlands, to measure scattering properties of irregularly shaped mineral aerosol samples ${ }^{2}$. This system was further developed at the IAA cosmic dust laboratory, Granada, Spain ${ }^{3}$. A comparison across these three setups, as well as an early version of our own system is described by Maconi et al. ${ }^{4,5}$. Lately, two more systems have been developed ${ }^{6,7}$. The latest one $^{7}$ allows characterization of levitated water droplets using a 100-kHz ultrasound levitator. As a light source, it uses a collimated, vertically polarized, continuous laser beam, with wavelength $\lambda=532.1 \mathrm{~nm}$ and laser power of $2 \mathrm{~W}$.

However, there was no experimental setup that can reliably measure light scattered by a fixed small particle with dimensions ranging between micrometer and millimeter sizes. The goal of our setup is nondestructive characterization of such particles by measuring light scattered by a levitating sample, whilst simultaneously controlling its 3D position and rotation, and recording large amounts of data.

\section{RESEARCH METHODS AND MATERIALS}

Based on our experience in ultrasound manipulation and precise optical interferometric devices and in light scattering, we assembled an interdisciplinary group of scientists to develop a fully automated, 3D scatterometer that can measure scattered light at different wavelengths from small particulate samples. The setup comprises: (a) the PXI Express platform to synchronously record data from several photomultiplier tubes (PMTs); (b) a motorized rotation stage to precisely control the azimuthal angle of the PMTs around $360^{\circ}$; (c) a versatile light source, whose wavelength, polarization, intensity, and beam shape can be precisely controlled, (d) an ultrasound based levitator using 400 ultrasound transducers working at 100 $\mathrm{kHz}$ central frequency and (e) a high speed camera with imaging system (Fig.1).

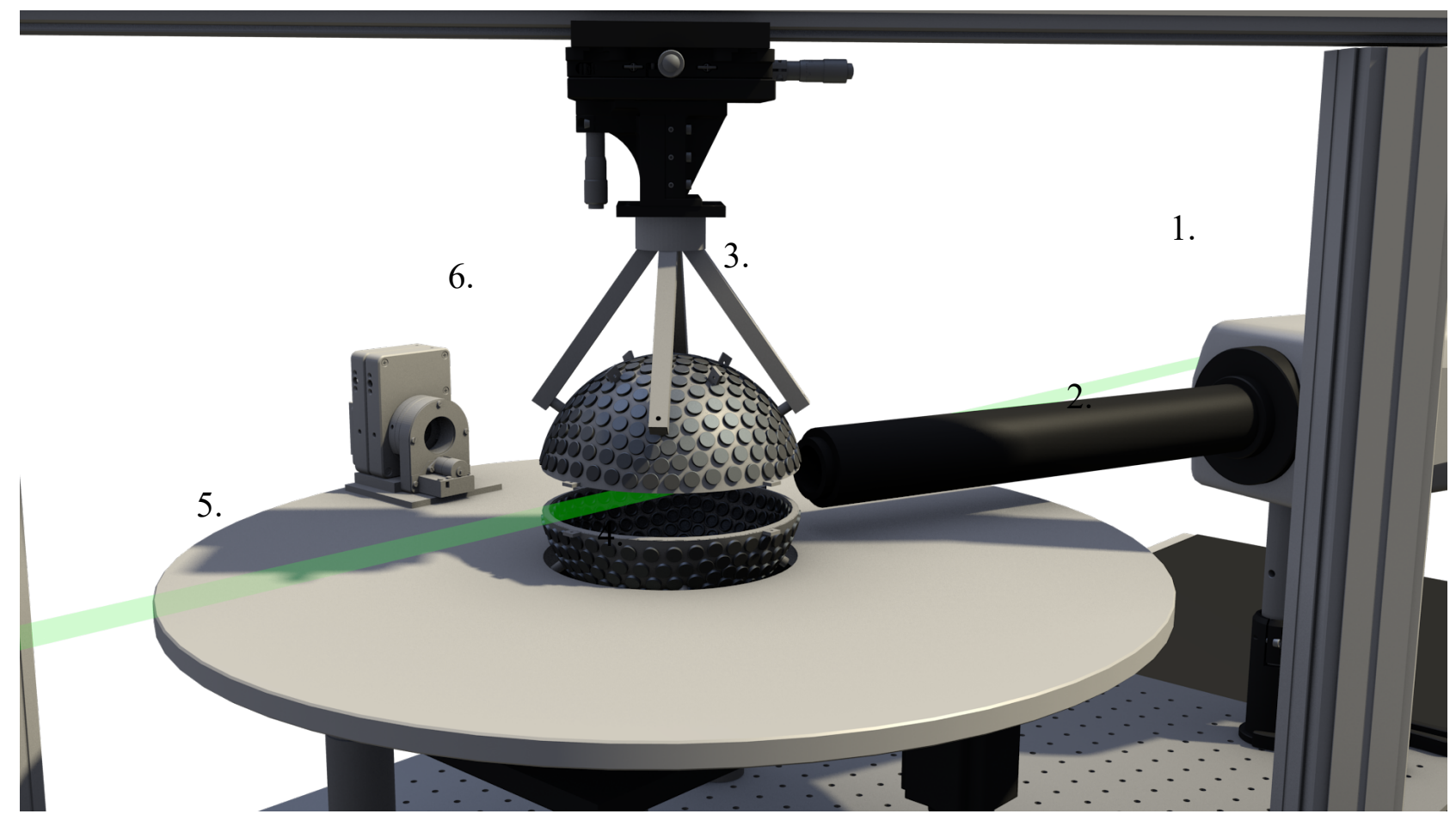

Figure 1. System Overview: 1. High-speed camera, 2. Imaging system, 3. Ultrasound based levitator, 4. Motorized rotation stage, 5. Light source output beam, 6. Measurement PMT. 


\subsection{Scattering theory}

We describe the polarization state of light beam using the Stokes parameters $I, Q, U$, and $V$, which we combine into a vector known as the Stokes vector. A scattered beam can thus be related to an incoming beam by multiplication with the $4 \times 4$ scattering matrix, $\mathbf{S}$. For an incoming vector $\boldsymbol{I}_{i}=\left(I_{i}, Q_{i}, U_{i}, V_{i}\right)^{T}$ and a scattered vector $\boldsymbol{I}_{s}=\left(I_{s}, Q_{s}, U_{s}, V_{s}\right)^{T}$ the transformation becomes:

$$
\boldsymbol{I}_{S}=\frac{1}{k^{2} R^{2}} \mathbf{S} \boldsymbol{I}_{\boldsymbol{i}}
$$

Here $R$ is the distance from the particle to the observer and $k$ is the wave number. Individual elements of the scattering matrix can be defined by measuring the scattered light at specific polarization states. To resolve all the matrix elements, the polarization of the incoming light and scattered light are controlled by a linear polarizer and a quarter wave plate ${ }^{5}$. With the linear polarizers only, our device can construct the upper left $2 \times 2$ submatrix of $\mathbf{S}$. The Mueller matrices for a linear polarizer $\mathbf{M}_{p}$ is:

$$
\mathbf{M}_{p}(\theta)=\frac{1}{2}\left(\begin{array}{cccc}
1 & \cos (2 \theta) & \sin (2 \theta) & 0 \\
\cos (2 \theta) & \cos ^{2}(2 \theta) & \sin (2 \theta) \cos (2 \theta) & 0 \\
\sin (2 \theta) & \sin (2 \theta) \cos (2 \theta) & \sin ^{2}(2 \theta) & 0 \\
0 & 0 & 0 & 0
\end{array}\right)
$$

Thus, for one single measurement with the scatterometer, the chained transformation becomes:

$$
\boldsymbol{I}_{s}=\mathbf{M}_{p}\left(\theta_{2}\right) \mathbf{M} \mathbf{M}_{p}\left(\theta_{1}\right) \boldsymbol{I}_{i},
$$

where $\mathbf{M}=k^{-2} R^{-2} \mathbf{S}$. The following configurations are needed to construct the $2 \times 2$ submatrix:

$$
\begin{gathered}
I_{1}: \theta_{1}=0^{\circ} ; \theta_{2}=0^{\circ} \\
I_{2}: \theta_{1}=90^{\circ} ; \theta_{2}=90^{\circ} \\
I_{3}: \theta_{1}=0^{\circ} ; \theta_{2}=90^{\circ} \\
I_{4}: \theta_{1}=90^{\circ} ; \theta_{2}=0^{\circ}
\end{gathered}
$$

From these measurements, the Mueller matrix elements can be calculated as:

$$
\begin{aligned}
& M_{11}=I_{1}+I_{2}+I_{3}+I_{4} \\
& M_{12}=I_{1}-I_{2}+I_{3}-I_{4} \\
& M_{21}=I_{1}-I_{2}-I_{3}+I_{4} \\
& M_{22}=I_{1}+I_{2}-I_{3}-I_{4}
\end{aligned}
$$

\subsection{Measurement setup}

The system utilizes a tunable multimode Argon-krypton laser (Melles Griot 35 KAP 431), with 12 wavelengths ranging from 465 to $676 \mathrm{~nm}$, with beam quality factor $\mathrm{M} 2=1.2$, and a power of 4 to $20 \mathrm{~mW}$, depending on wavelength, linear polarizers (Thorlabs LPVISE100-A), a reference PMT monitoring beam intensity, and a measuring PMT (Hamamatsu microPMT H12403-01) mounted radially from the sample at adjustable radii. The PMT signal is amplified in a Thorlabs TIA60 transimpedance amplifier and captured by an NI PXIe-5171R oscilloscope module. The current $300 \pm 10$-mm radius allows measuring all azimuthal angles except for $\pm 11^{\circ}$ around the backward scattering direction. A rotation stage (Standa 8MRB240-152-59D) controlled through the NI PXIe-8880 moves the measurement head, comprising IR filter, shutter, polarizer and a PMT. The measurement angle is controlled by the motor-driven rotation stage with an accuracy of 15 ' (Fig. 2).

To reduce external light and reflections, the device is enclosed inside three adjacent chambers covered with a black light absorbing material. The first chamber contains beam shaping and filtering optics, the second contains the levitator and measurement PMT placed on the motorised rotation stage, whereas the third chamber contains the beam stop. The laser is outside the enclosure for improved temperature control. The temperature and relative humidity in all chambers are recorded 
during the measurements. The light is transferred from the laser by a polarization-maintaining fiber into the first chamber, where the light is collimated.

The sample is placed into the levitator. The acoustic levitator is an array of circa 400 ultrasonic transducer placed on two separated hemispheres with the intended levitation spot in the centre. The array is divided into 24 sections whose amplitudes and phases can be controlled. The transducers (MCUST10P40B07RO, Multicomp) have a diameter of $10 \mathrm{~mm}$ and central frequency of $40 \mathrm{kHz}$. The amplitudes and phases are adjusted in accordance with the principle of the acoustic Gor'kov potential ${ }^{7}$. This enables the levitation of particles in pressure nodes of the standing waves. The system can be used to levitate object for sufficient for the measurement amount of time (even for hours at the time, if there is a need).

A high speed camera (Phantom V611) is used to image the samples while levitated. Due to small size of the samples $(\sim 2$ $\mathrm{mm}$ and less) and the fact that placing the objective inside the spherical levitator (radius $\sim 90 \mathrm{~mm}$ ) is not possible a high magnification long working distance system is needed. Navitar 12X Zoom Lens (1-50487AD) enables 108mm working distance and so placing the objective outside of the levitation volume. Because of the high magnification and high framerate a proper illumination is needed. The sample is illuminated from two directions with IR LEDs (850nm). During the scattering measurement the illumination is turned off.

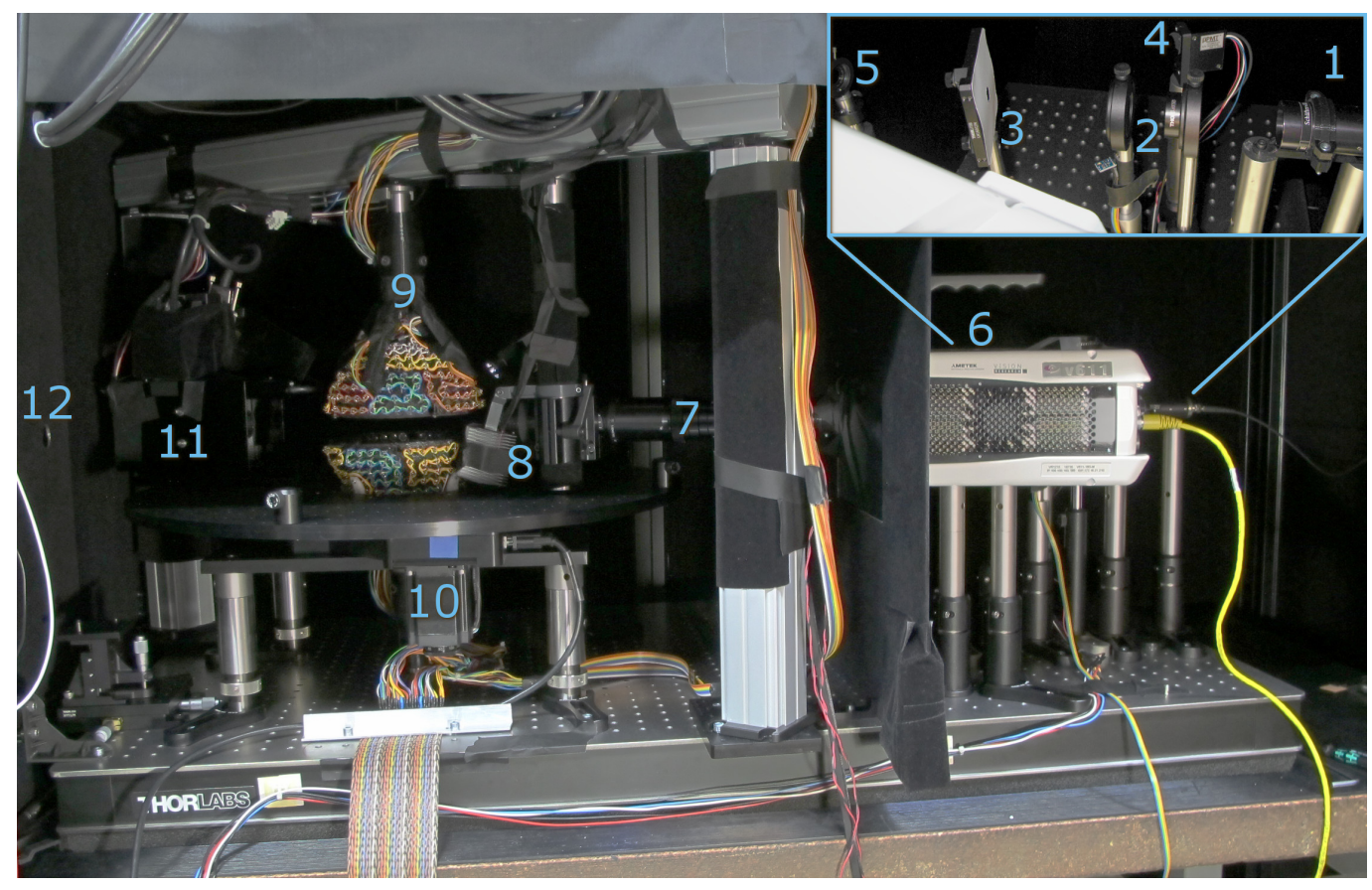

Figure 2. Detailed view of the instrument. (1) Collimator. (2) Polarizer for incoming beam. (3) Diffuse reflector with aperture. (4) Reference PMT. (5) Adjustable iris. (6) High-speed camera. (7) Imaging optics. (8) LED illumination for imaging. (9) Orientation-controlled acoustic levitator. (10) Motorized rotating breadboard. (11) Measurement head, comprising IR filter, shutter, polarizer and PMT. (12) Beam stop aperture.

\subsection{Alignment of the system}

Before performing calibration measurements in the case of static measurements, when the sample $3 \mathrm{~mm}$ glass sphere is placed on the holder the system was aligned according the procedure described by Maconi et al. ${ }^{4}$.

In the case of levitated sample the calibration procedure was done by first aligning a needlelike pressure sensor in the center of the system following the former procedure. This pressure sensor was now used to calibrate the levitator array such that the levitation spot would be in the position of needles tip. After inserting the levitated sample it was inspected that the shadow was close to center of the beam. 


\section{RESULTS}

To calibrate our system, we measured a clear glass sphere ( $\mathrm{d}=3 \mathrm{~mm}, \mathrm{~N}-\mathrm{BK} 7$ glass, Edmund optics), using a 488-nm laser light. We use four different polarization configurations to obtain four elements of the Mueller matrix. Figure 3 shows results for the vertical-vertical, horizontal-horizontal vertical-horizontal, and horizontal-vertical configurations of the polarizers. Furthermore, we modelled the scattering properties of clear glass spheres with the abovementioned parameters. During the above mentioned measurements the sample (glass sphere) was placed onto a holder as described by Maconi et al. ${ }^{4}$.

In the second stage of our calibration measurements we levitated the same sphere and used the same configurations of the polarizers. The results from modeling and measurements are presented in Fig. 4.

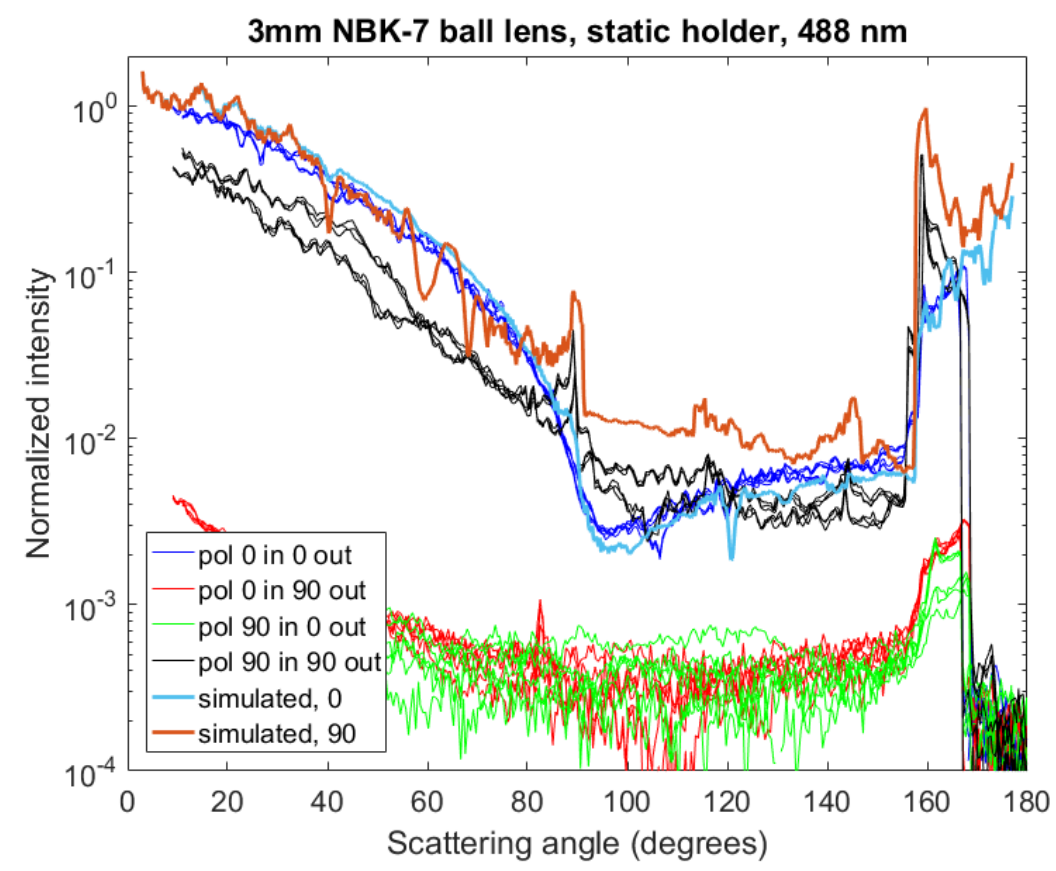

Figure 3. Measured light scattering intensity of a 3-mm glass sphere on a static holder. Polarizers configured: vertical-vertical, horizontal-horizontal, vertical-horizontal, and horizontal-vertical polarizations.

One candidate for a realistic mineral sample that can be modeled theoretically was agglomerated microscopic silica particles. The silica dust was produced by Sigma-Aldrich and contains irregular particles in the size range $0.5-10 \mu \mathrm{m}$, which have a natural tendency to clump together. A roughly ellipsoidal piece of agglomerate was selected for our sample, and measured while held in place by the acoustic levitator (Fig. 5).

\section{DISCUSSION}

To eliminate disturbances produced by the sample holder during the calibration and sample measurements was always a challenge. Nevertheless, after modifications, as well as taking into account the results obtained without the sphere, we were able to compensate for the scattered light from the holder.

To fine-tune the levitator and verify the stability of our sample, we image the sample before every measurement. However, the camera lens and IR LED source were producing significant light scattering artifacts, even with strong filtering of the IR light. To solve this problem, the IR LED sources were moved out of the way and the camera lens covered with a black velvet lid before measurement. This was enough to reduce stray scattering artifacts to below our detection level. 


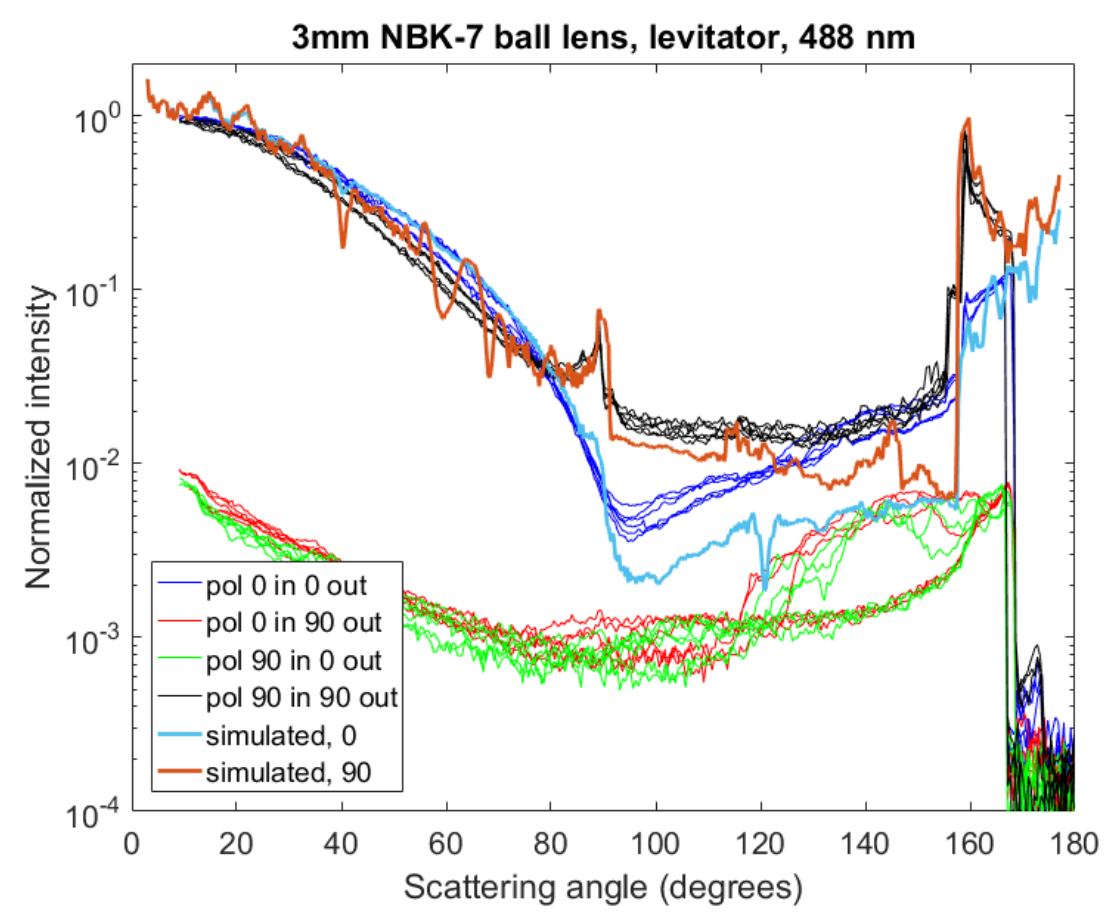

Figure 4. Measured light scattering intensity of a levitated 3-mm glass sphere. Polarizers configured: vertical-vertical, horizontal-horizontal, vertical-horizontal, and horizontal-vertical polarizations.

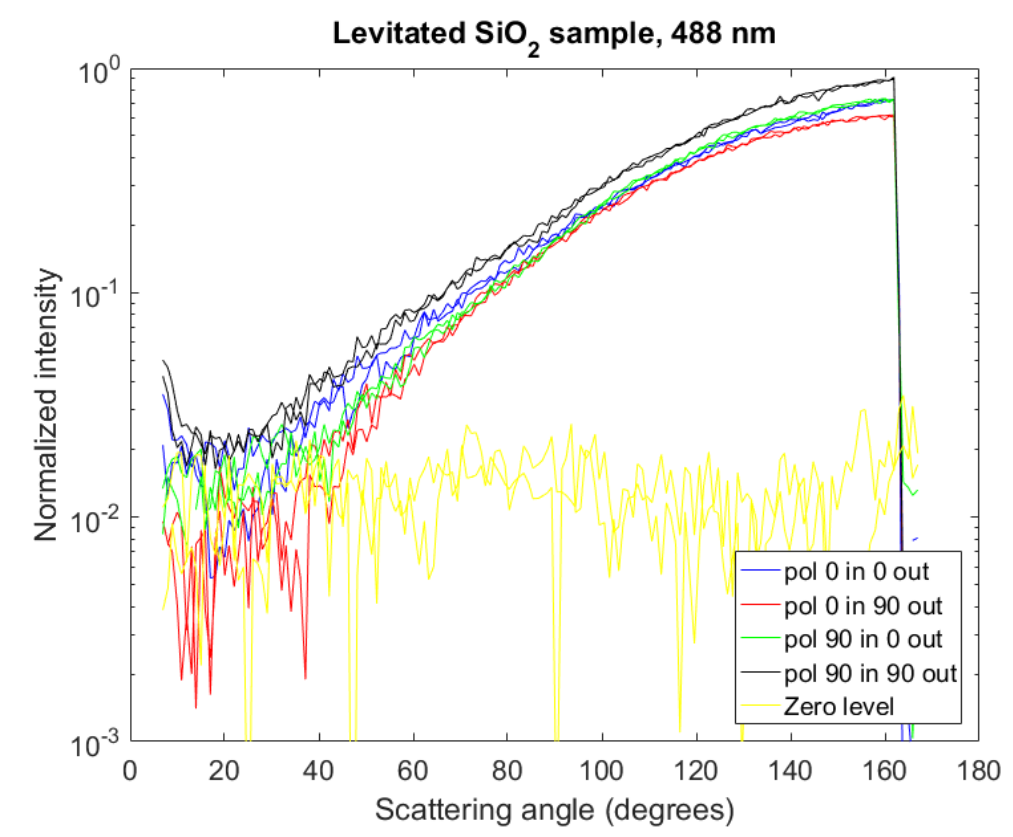

Figure 5. Measured light scattering intensity of a levitated orientation-controlled silica sample. Light intensity was scanned on the right side of the sample, which was oriented with its major axis parallel to the incoming beam. The sample was also measured with no incoming light, to assess the amount of ambient light scattered. 
The laser currently emits vertically polarized light only. Horizontal polarization is achieved by transmitting the beam through a polarization-maintaining fiber and using a quarter-wave plate at $45^{\circ}$ angle to convert the light to circular polarization, allowing the operator to easily choose any polarization direction with a linear polarizer.

\section{CONCLUSIONS}

Our scatterometer is a valuable tool for measuring optical properties of levitated small particles and helps us validate and prove theoretical models. It also allows us to collect data to gain new theoretical understanding about optical properties of both individual and groups of small particles, thus fulfilling the main goal of the project.

The device is the first of its kind, since it measures spectral angular scattering, at different polarizations, for an arbitrary object in the $\mu \mathrm{m}$-mm size scale. It permits a non-destructive, disturbance-free measurement with control of the orientation and location of the scattering object.

\section{ACKNOWLEDGEMENTS}

This study was conducted under the support from the European Research Council, in the frame of the Advanced Grant project No. 320773 'Scattering and Absorption of Electromagnetic Waves in Particulate Media' (SAEMPL).

\section{REFERENCES}

[1] Hunt, A. J. and Huffman, D. R., “A new polarization-modulated light scattering instrument,” Review of Scientific Instruments 44(12), 1753-1762 (1973).

[2] Volten, H., Munoz, O., Rol, E., Haan, J. d., Vassen, W., Hovenier, J., Muinonen, K., and Nousiainen, T., "Scattering matrices of mineral aerosol particles at $441.6 \mathrm{~nm}$ and $632.8 \mathrm{~nm}$, , Journal of Geophysical Research: Atmospheres 106(D15), 17375-17401 (2001).

[3] Muñoz, O., Moreno, F., Guirado, D., Ramos, J., López, A., Girela, F., Jerónimo, J., Costillo, L., and Bustamante, I., "Experimental determination of scattering matrices of dust particles at visible wavelengths: The IAA light scattering apparatus," Journal of Quantitative Spectroscopy and Radiative Transfer 111(1), 187-196 (2010).

[4] Maconi, G., Kassamakov, I., Penttilä, A., Gritsevich, M., Hæggström, E., and Muinonen, K., "Experimental light scattering by small particles: System design and calibration," in [Optical Measurement Systems for Industrial Inspection X], Proc. SPIE 10329, 103292S, International Society for Optics and Photonics (2017).

[5] Maconi, G., Penttilä, A., Kassamakov, I., Gritsevich, M., Helander, P., Puranen, T., Salmi, A., Hæggström, E., and Muinonen, K., "Non-destructive controlled single-particle light scattering measurement," Journal of Quantitative Spectroscopy and Radiative Transfer 204, 159-164 (2018).

[6] Onofri, F. R., Krzysiek, M. A., Barbosa, S., Messager, V., Ren, K.-F., and Mroczka, J., "Near-critical-angle scattering for the characterization of clouds of bubbles: particular effects," Applied optics 50(30), 5759-5769 (2011).

[7] Onofri, F. R., Ren, K. F., Sentis, M., Gaubert, Q., and Pelcé, C., "Experimental validation of the vectorial complex ray model on the inter-caustics scattering of oblate droplets," Optics express 23(12), 15768-15773 (2015).

[8] Gor'kov, L., "On the forces acting on a small particle in an acoustical field in an ideal fluid," in [Soviet Physics Doklady], 6, 773 (1962). 